\title{
Cowper's Glands Abscess with Spreading to Adjacent Organs and Tissues with Development of Septic Shock: An Extremely Rare Case
}

\section{Zieratsho Kadyrov \\ Vladimir Stepanov \\ Emilbek Aldyrakov \\ Shota Ramishvili}

Department of Endoscopic Urology, Peoples' Friendship University of Russia (RUDN University), Moscow, Russian Federation
Correspondence: Zieratsho Kadyrov Email zieratsho@yandex.ru
Background: We present here the first case of Cowper's gland abscess complicated by septic shock and breakthrough of the abscess into the paraurethral region, cavernous body, scrotum, and pararectal tissue.

Case Presentation: A 63-year-old patient was admitted with complaints of temperature increase up to $39^{\circ} \mathrm{C}$, pain and enlargement of the perineum and the right half of the scrotum, frequent and difficulty urination, weakness, dizziness, dry mouth, and a sharp deterioration of the general condition. Clinical-laboratory data showed the presence of septic shock with unstable haemodynamics and many concomitant diseases. From the history, it is known that the patient for more than 20 years suffered from urinary tract infection and urinary disorders. Six months earlier, the patient underwent a puncture of a bulbourethral abscess. According to the ultrasound of the scrotum, TRUS, and MRI, bulbourethral abscess with spread to the right half of the scrotum, a part of the cavernous body, and the cellular tissue of the left sciatic-rectal fossa was diagnosed. Purulent cavities were opened with two incisions and drained. A cystostomy was installed. In the intensive care unit, according to a microbiological study (Escherichia coli, Klebsiella pneumoniae, and Klebsiella pneumoniae), antibacterial, detoxification, and symptomatic therapy of concomitant diseases were carried out together with a resuscitator and therapist. The patient was discharged on the 30th day with a negative analysis of urine culture, with a cystostomy, which was removed six months after the independent restoration of urination and closure of the fistulous passage between the urethra and Cowper's glands.

Conclusion: Untimely treatment of the very rare abscess of the Cowper's gland can lead to serious complications, up to the spread of a purulent process to neighbouring organs and tissues, and the development of septic shock, which will require urgent and intensive therapy with the involvement of experts from interdisciplinary fields.

Keywords: Cowper's glands abscess, bulbourethral abscess, infection, cowperitis, septic shock, complications

\section{Background}

Cowper's glands, or bulbourethral glands, are an accessory genital organ whose ducts drain into the bulbous urethra. ${ }^{1}$ The gland and its duct can be affected by both congenital and acquired diseases. They can be asymptomatic or cause difficulty in urinating or urinary retention. ${ }^{2}$ Among congenital pathologies, some scientists distinguish syringocele, which is today diagnosed and easily corrected endoscopically. ${ }^{1,3}$ Among the acquired diseases of Cowper's glands, cases of 
acute and chronic cowperitis, calcification in elderly patients, and tuberculous lesions of the glands have been described. ${ }^{1,4,5}$ However, there can be found only a few descriptions of such a rare disease as Cowper's gland abscess, or bulbourethral abscess in the recent works, which raise the interest in this problem among urological specialists. ${ }^{4,6,7}$ In this article, we describe an extremely rare case of Cowper's gland abscess complicated by septic shock, and breakthrough of the abscess mass into adjacent organs and tissues. Such cases with the development of serious complications require emergency measures both in terms of diagnosis and treatment. ${ }^{8,9}$

\section{Case Presentation}

H., 63 years old, was admitted to the clinic with complaints of fever up to $39^{\circ} \mathrm{C}$, pain and enlargement of the perineum and the right half of the scrotum, frequent and difficulty urination, weakness, dizziness, dry mouth, decreased urine output, deterioration of the general condition. Relatives were involved in collecting complaints and clarifying the anamnesis due to the severity of the condition. Medical history: six months ago the patient was discharged from another clinic, where a puncture of a bulbourethral abscess was performed. Besides, the patient for more than 20 years suffered from urinary tract infection and urinary disorders. As advised by a doctor, the patient periodically took antibiotics and alpha1-blockers. The patient has previously been repeatedly treated for urinary tract infections caused by Chlamydia trachomatis and Ureaplasma urealyticum. E. coli $\left(10^{5} \mathrm{CFU} / \mathrm{mL}\right)$ and Staphylococcus saprophytes $\left(10^{4} \mathrm{CFU} / \mathrm{mL}\right)$ were determined in the analyses of urine culture. Serum PSA tests were normal.

Concomitant chronic diseases: ischemic heart disease, permanent form of atrial fibrillation; chronic heart failure IIB; dilated cardiomyopathy; cerebrovascular disease; chronic cerebral ischemia with vertebrobasilar insufficiency; hypertension of the 3rd stage, risk of CVC (cardiovascular complications)- IV.

The objective condition on admission was severe. The patient was inhibited, disoriented, speech contact was difficult. The skin was pale and with a marbled shade. The body temperature was $39^{\circ} \mathrm{C}$, pulse rate -98 beats $/ \mathrm{min}$ with an irregular rhythm, blood pressure - 85/40 mmHg. Respiratory rate - 24 breaths per minute. The kidney area was painless on palpation. Urination was difficult and frequent. The bladder was not detected by percussion and palpation. The prostate gland was painful on palpation, enlarged, in places firm, without areas of fluctuation. The external genital organs formed correctly. The right half of the scrotum was enlarged, the skin of the scrotum was hyperaemic, there was oedema in the area of the bottom of the scrotum on the right, the skin was thinned, there were areas of fluctuation. The right testicle was not enlarged, painful on palpation, of dense consistency, with a smooth surface. The left half of the scrotum was normal. In the perineum, there was oedema and swelling, painful on palpation, mild infiltration along the urethra, which was not palpable. In the left gluteal region, there was oedema, skin hyperaemia, and soreness. The general blood test showed the following results: hematocrit $-38.3 \%$, leukocytosis $-30.1 \times 10^{9} / 1$, segmented neutrophils $-60 \%$, stab neutrophils - $20 \%$, lymphocytes - $17 \%$, blood sugar - 5.6 $\mathrm{mmol} / \mathrm{mL}$. In the general analysis of urine, pronounced leukocyturia was determined, urine was cloudy. Urine was taken for culture.

From the emergency room, after examination by a urologist, proctologist, therapist, and resuscitator, due to hypotension and for further stabilization of haemodynamics, the patient was transferred to the intensive care unit, where appropriate therapy (infusion therapy with vasopressors) was carried out.

After the stabilization of haemodynamics, an examination of the patient was carried out. According to the ultrasound data, the kidneys and bladder were unchanged (urine volume in the bladder - $50 \mathrm{~mL}$ ). The ultrasound of the scrotal organs has revealed the following: the testes were intact, an anechoic paratesticular formation of $40 \times 34 \mathrm{~mm}$ in size was situated in the right half of the scrotum (Figure 1). According to the TRUS data, the prostate gland had a smooth contour, with heterogeneous echo structure and hyperechoic areas in the paraurethral zone of about $12 \mathrm{~mm}$ (calcifications). The gland's hypoechoic area was about $10 \mathrm{~mm}$ in the transient zone. The volume of the gland was $38 \mathrm{~cm}^{3}$ (Figure 2). On the ultrasound, a hypoechoic area of $68 \times 35 \mathrm{~mm}$ was noted paraurethrally below the pelvic diaphragm (Figure 3). Based on the MRI analysis of the pelvic organs, a paraurethral abscess spreading to the right half of the scrotum and the tissue of the left sciatic-rectal fossa, heterogeneity of the transient part of the prostate gland, infiltration, and an affected area of the corpus cavernosum, paraprostatic tissue, and mesorectal fascia, and lymphadenopathy were observed (Figure 4A and B). In the biochemical analysis of blood, attention was drawn to an increase in the level of blood creatinine to $193 \mu \mathrm{mol} / \mathrm{L}$, of urea to $10 \mathrm{mmol} / \mathrm{L}$, of 


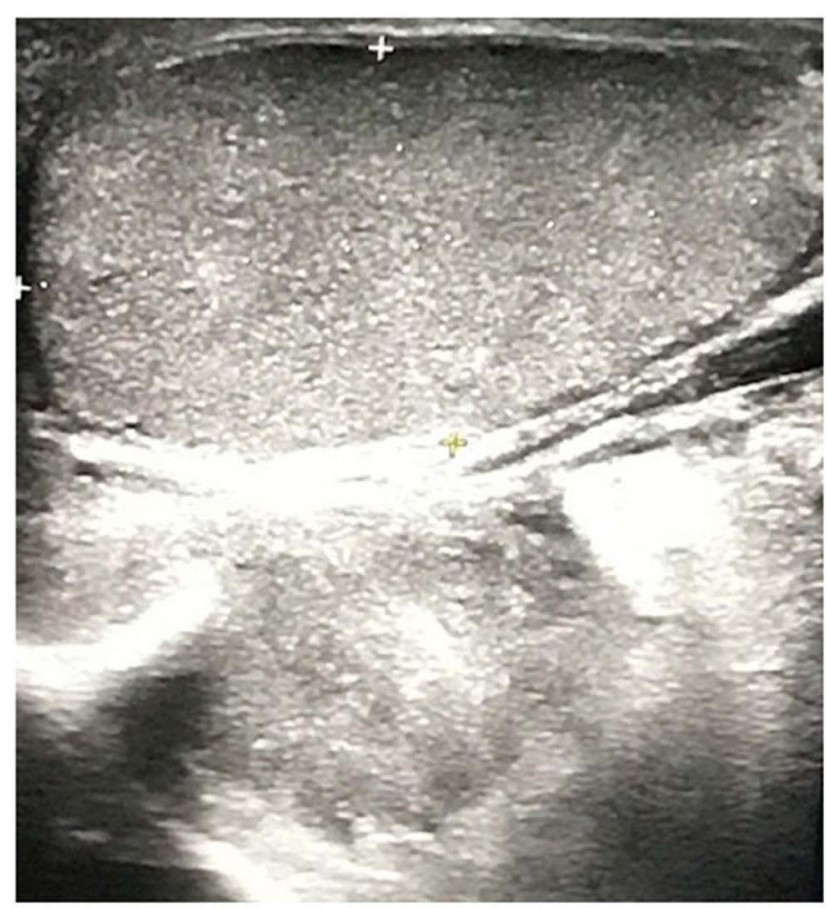

Figure I Ultrasound image of the scrotum. The right testicle is not changed. Paratesticular anechoic formation of $40 \times 34 \mathrm{~mm}$ with irregular contours can be found in the tunics of the scrotum.

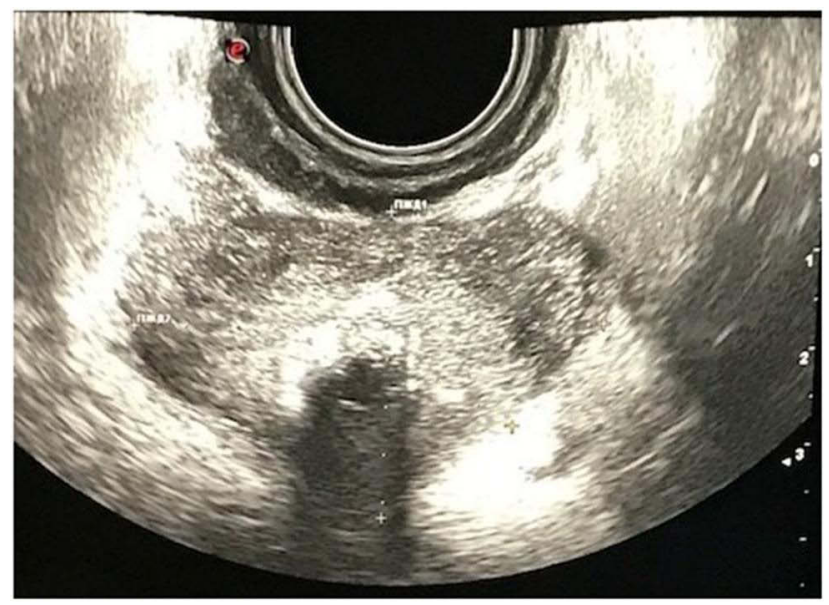

Figure 2 TRUS data: the prostate gland with a smooth contour, of heterogeneous echo structure, with hyperechoic areas in the paraurethral zone of about $12 \mathrm{~mm}$ (calcifications) and an acoustic path. The volume of the gland is $38 \mathrm{~cm}^{3}$.

procalcitonin to $6.8 \mathrm{ng} / \mathrm{mL}$, and of CRP to $178 \mathrm{mg} / \mathrm{L}$. Acid-base balance was the following: $\mathrm{pH}$-value - 7.25, base deficit - $9.2 \mathrm{mmol} / \mathrm{l}$; partial pressure for $\mathrm{CO}_{2}$ $32 \mathrm{~mm} \mathrm{Hg}$, and for $\mathrm{O}_{2}-58 \mathrm{~mm} \mathrm{Hg}$. Diuresis was about $400 \mathrm{~mL}$ per day (according to relatives).

On an emergency basis, three hours after the hospitalization, the patient underwent surgical treatment: a purulent cavity was opened by a midline scrotal-

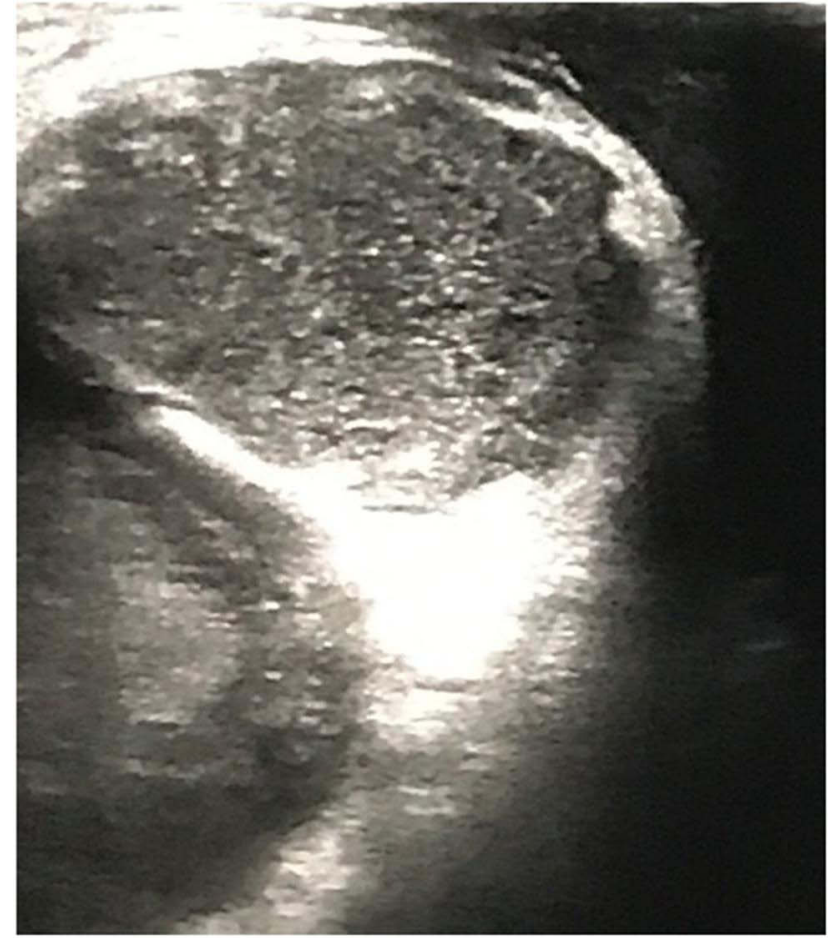

Figure 3 Ultrasound of the perineum: paraurethrally, below the pelvic diaphragm, a hypoechoic oblong area of $6.8 \times 3.5 \mathrm{~cm}$ is observed.

perineal incision (Figure 5A) and about $120 \mathrm{~mL}$ of yellow material was evacuated. The spread of the pus reached the pelvic diaphragm. The urethra and corpus cavernosum were infiltrated and in one area, a purulent lesion of the cavernous body of about $1 \mathrm{~cm}$ was revealed, which was then opened (Figure 5B). Pus was not detected during the puncture of the central lobe of the prostate. A separate incision opened a cellular space of the pelvis in the left sciatic-rectal fossa (Figure 5C), where about $50 \mathrm{~mL}$ of pus was also evacuated. A cystostomy was installed after (Figure 5D).

According to the results of a microbiological study, Escherichia coli and Klebsiella pneumoniae were detected in the urine culture. Moreover, the growth of Klebsiella pneumoniae was determined from the wound discharge. Based on these results, antibacterial therapy was prescribed. Subsequent positive dynamics were noted in the form of a decrease in body temperature, a decrease in leukocytosis, CRP, and procalcitonin levels, in normalization of creatinine and urea levels, and stabilization of hemodynamic parameters. On the 11th day, the patient was transferred from the intensive care unit to the urology department for further treatment. Retrograde urethrocystography revealed leakage of contrast agent in the bulbous 

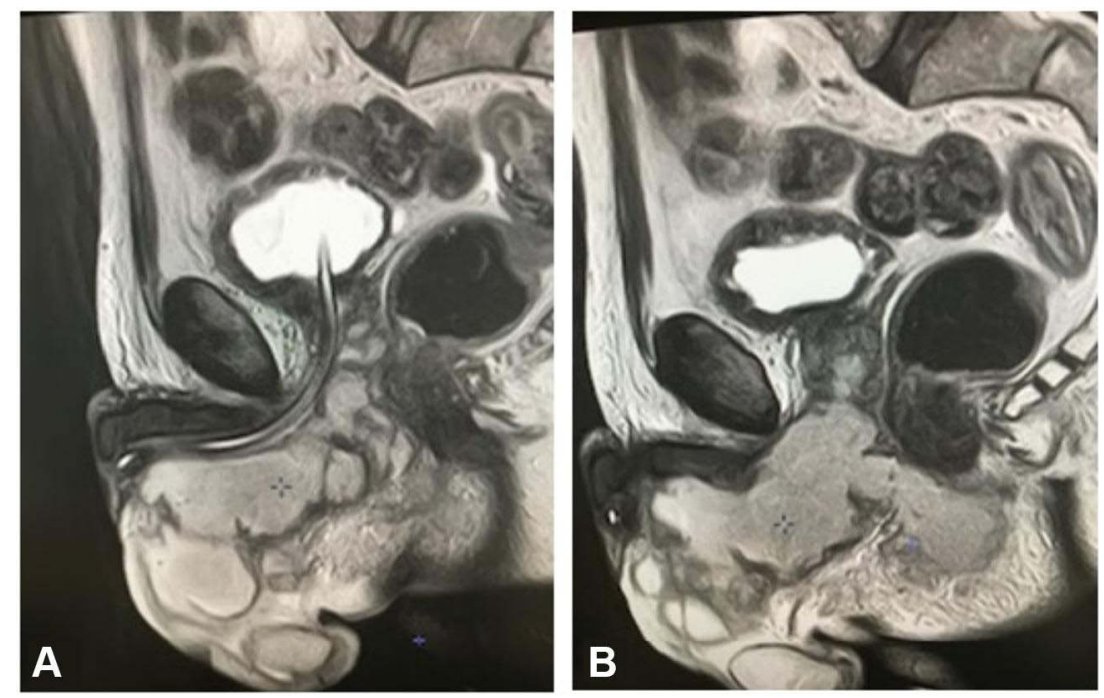

Figure 4 MRI of the pelvis: (A and B) paraurethral abscess spreading to the right half of the scrotum, to the cellular tissue of the left sciatic-rectal fossa, heterogeneity of the transient part of the prostate gland, and infiltration of paraprostatic tissue and mesorectal fascia.

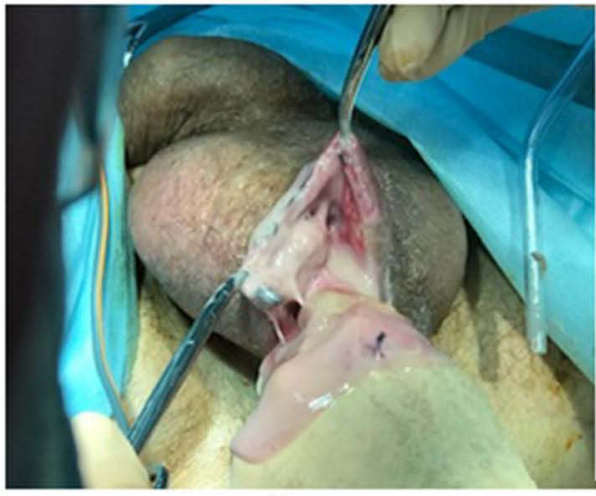

A

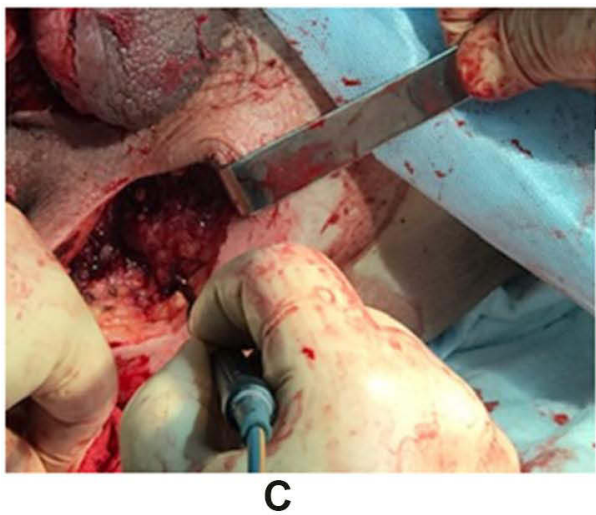

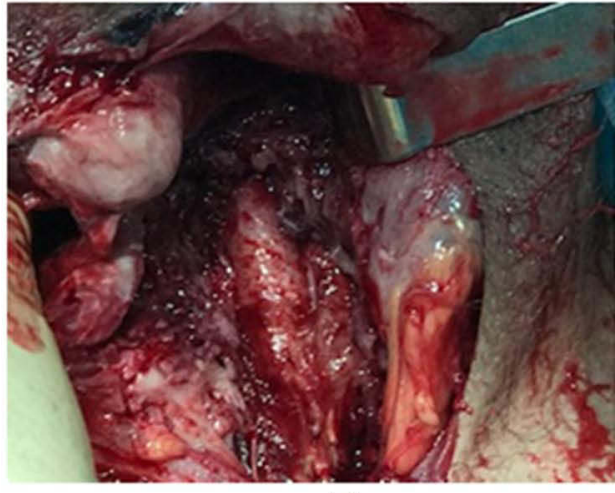

B

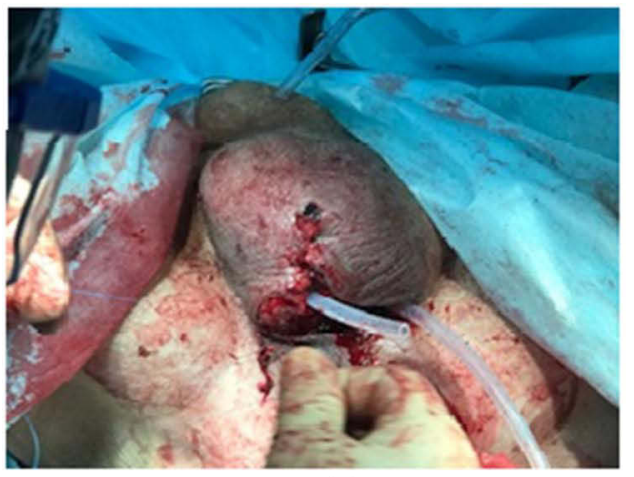

D

Figure 5 Stages of the operation: (A) opening the abscess by a median scrotal-perineal incision; (B) wound after evacuation of pus from the scrotum and paraurethral region, the urethra is intact; (C) opening the purulent cavity of the left sciatic-rectal fossa on the left; (D) drainage of the paraurethral region and scrotal wound.

urethra (Figure 6), which confirmed the presence of a fistula between the urethra and Cowper's glands but excluded syringocele. ${ }^{2,4}$ The patient was transferred to the therapeutic department for further treatment of concomitant diseases and was discharged home on the 30th day after the surgery with a negative urine culture, with a cystostomy, which was removed six months after spontaneous restoration of urination and closure of the 


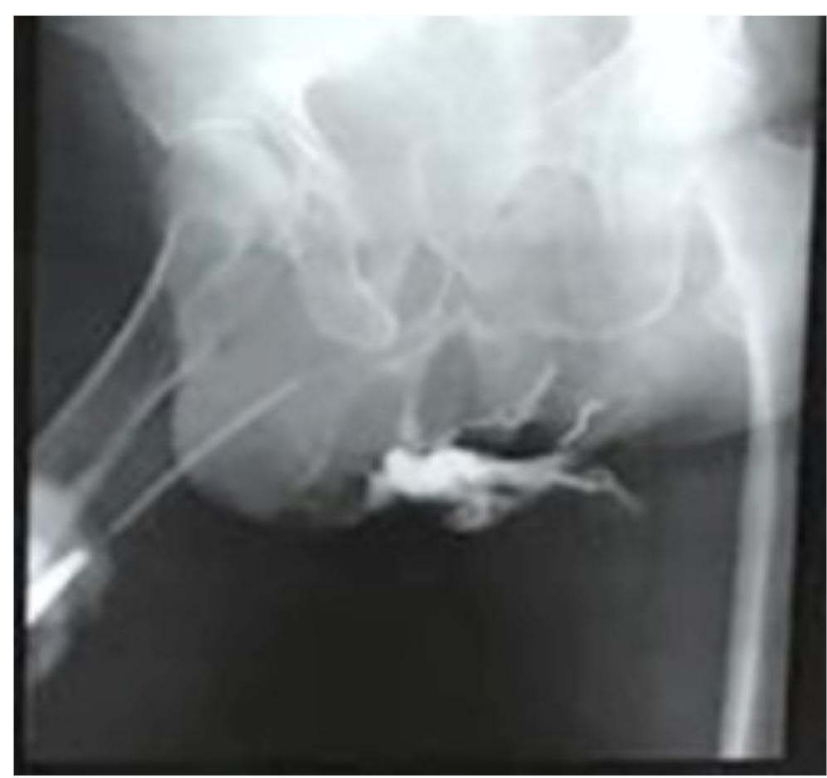

Figure 6 Urethrogram on the 14th day after surgery. Contrast agent leaks into the perineum and pelvic cellular tissue.

fistulous passage between the urethra and Cowper's glands.

\section{Discussion and Conclusions}

The problem of the appropriate treatment of the inflammatory process with the development of abscess of the bulbourethral, or Cowper's, glands is of particular interest today because it is detected very rarely. ${ }^{1,3}$ The inflammatory process is most often associated with infections of the genitourinary system (Escherichia coli, Pseudomonas aeruginosa, Neisseria gonorrhoea, and Chlamydia trachomatis). ${ }^{1,3-7}$ Besides, a provoking factor in the development of cowperitis and bulbourethral abscesses in men is prostate biopsy or transurethral interventions. The main clinical manifestations of such conditions are high body temperature, pain in the perineal region, and stranguria. ${ }^{4,6,7}$ For instrumental confirmation of the presence of a paraurethral abscess, for differential diagnosis of complications in the form of syringocele, and to exclude the presence of a fistulous passage from adjacent cellular spaces of the pelvis, ultrasound of the perineum and small pelvis, ascending urethrography, and MRI of the pelvic organs should be performed. $^{3-5}$ The treatment of bulbourethral abscesses consists of a puncture and drainage of a purulent focus with the prescription of antibacterial agents according to urine culture and the contents of the purulent cavity. 1,6,7
The literature describes the only case of the development of a bulbourethral abscess with the development of sepsis in a patient who was diagnosed with an abscess recurrence in more than one year and successfully treated with transperineal surgical puncture and intensive antibiotic therapy. ${ }^{6,7}$

The development of septic shock with unstable haemodynamics in patients with the presence of a purulent focus remains a life-threatening condition and requires immediate measures, including accurate diagnosis of the localization and extent of the spread of the purulent process, assessment of the state of vital organs, stabilization of haemodynamic parameters, sanitation and drainage of a purulent focus, intensive therapy maintenance of vital organs and prevention of complications. ${ }^{8-11}$

As shown in this study, with the development of septic shock, symptoms of intoxication begin to predominate and manifest themselves up to unstable haemodynamics in the clinical picture. In septic conditions, in addition to neutrophilic leukocytosis with a shift in the leukocyte formula to the left and acid-base imbalance, there is an increase in markers of systemic inflammatory response in the blood (CRP, procalcitonin) in laboratory tests.

The presented clinical case is the first manifestation of a bulbourethral abscess with the development of septic shock, where a patient with a long history of urinary infection, inadequate treatment, and a late visit to the doctor against the background of many concomitant diseases developed a bulbourethral abscess with complications. The analysis of the case showed that the abscess occurred due to a urinary tract infection caused by Escherichia coli and Klebsiella pneumoniae. Probably, an important role was also played by the STIs caused by Chlamydia trachomatis and Ureaplasma urealyticum, which were present in the organism for a long time.

In the presented case, the spread of the purulent process corresponded to the topographic anatomy and the path of the spread of urinary leaks and pus in case of damage to the urethra and paraurethral tissue (Figure 7A). At the same time, the purulent process and infiltration were detected in the corpora cavernosa of the penis, and were not detected in the hypogastric region, but spread into the cellular space of the pelvis (Figure 7B), which is not typical for urinary and purulent intrusions and indicates severe damage to the surrounding tissues and organs.

The success in the treatment of a patient with a widespread bulbourethral abscess and the development of septic shock has been achieved through the implementation of therapeutic and diagnostic measures in the first 


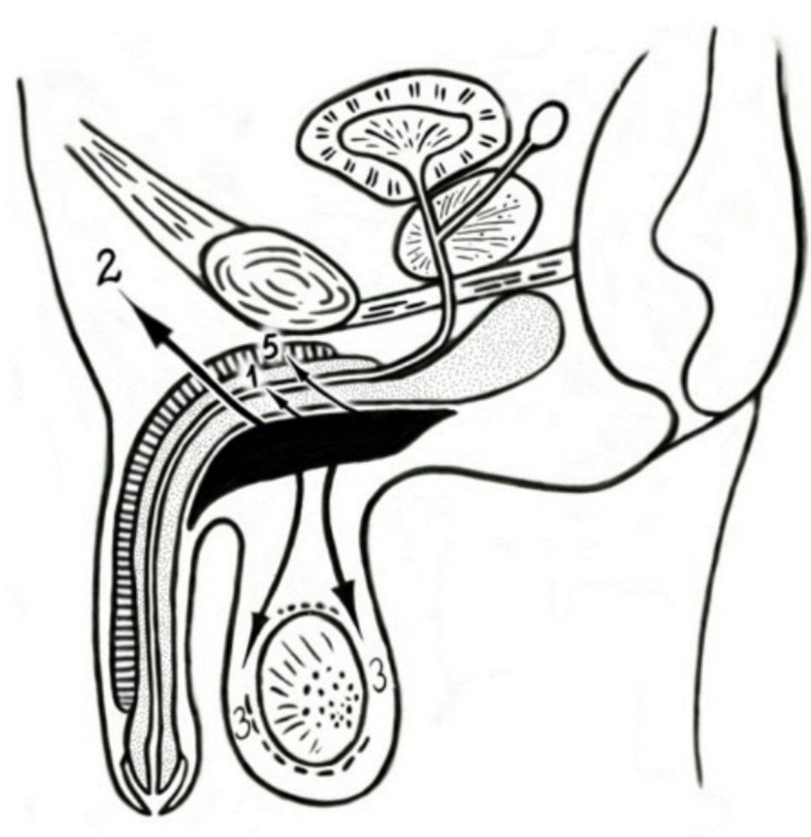

A

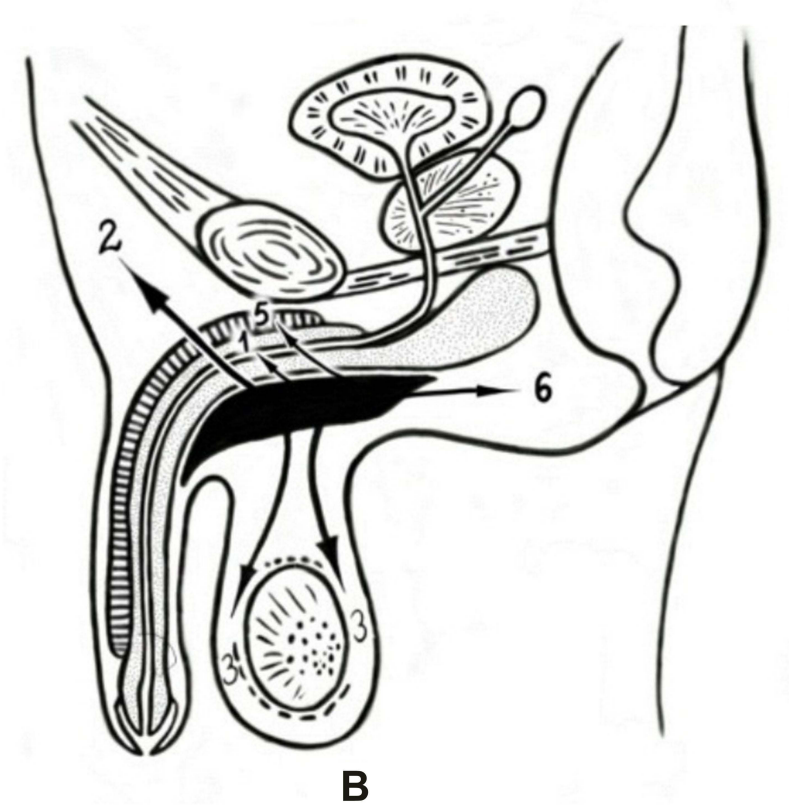

Figure 7 (A) Schematic presentation of the spread of urinary leakage and pus in the surrounding organs and tissues from the paraurethral region. (B) Schematic presentation of the spread Cowper's abscess to the surrounding organs and tissues: I - to the urethra; 2 - in the hypogastric region; 3- into the scrotum; 4- in the perineum (paraurethrally); 5 - into the corpora cavernosa; 6- to the pararectal space.

hours of hospital stay in accordance with the recommendations: the rapid recovery of haemodynamic disbalance, maintenance and control of impaired vital functions, early detection and sanitation of the source of generalization of the infection - within no more than six hours after the diagnosis, early and rational antimicrobial therapy (empirical, then according to the culture after the earliest identification of the pathogen). ${ }^{8-11}$ Besides, general treatment (prevention of thromboembolic complications, pressure and acute ulcers, nutritional support, and symptomatic treatment), control of metabolism, and normalization of metabolic constants (glycemia, blood $\mathrm{pH}$, blood lactate, saturation of central venous blood, osmolarity, blood plasma electrolytes), and prevention of complications (terminal failure of organs and systems, re - and superinfection), carried out in the postoperative period, allowed to achieve the positive result.

Given the severe course of the bulbourethral abscess and the presence of many concomitant diseases, the treatment and discharge of the patient in a satisfactory condition were possible through the prompt involvement of related experts. This made it possible to adequately assess the situation, patient's condition, stabilize haemodynamics and conduct additional examinations, determine the prevalence of the purulent process and concomitant diseases, establish the final diagnosis and, finally, provide surgical, then conservative treatment.

\section{Abbreviations}

$\mathrm{CFU}$, colony-forming unit; CRP, c-reactive protein; CVC, cardiovascular complications; MRI, magnetic resonance imaging; PSA, prostate-specific antigen; STI, sexually transmitted infection; TRUS, transrectal ultrasound.

\section{Data Sharing Statement}

All data generated or analysed during this study are included in this published article.

\section{Consent for Publication}

The patient signed consent for publication of data (including individual details and images).

\section{Acknowledgments}

The publication has been prepared with the support of the "RUDN University Program 5-100".

\section{Author Contributions}

All authors contributed to data analysis, drafting or revising the article, gave final approval of the version to be published, and agree to be accountable for all aspects of the work. All the authors have read and approved the manuscript, and have agreed both to be personally accountable for the author's own contributions and to ensure that questions related to the accuracy or integrity of any part of 
the work, even ones in which the author was not personally involved, are appropriately investigated, resolved, and the resolution documented in the literature.

\section{Funding}

This study did not receive any specific grants from any funding agencies in the public, commercial, and non-profit sectors.

\section{Disclosure}

The authors declare that they have no competing interests.

\section{References}

1. Chughtai B, Sawas A, O’Malley RL, Naik RR, Ali Khan S, Pentyala S. A neglected gland: a review of Cowper's gland. Int J Androl. 2005;28:74-77. doi:10.1111/j.1365-2605.2005.00499.x

2. Colodny AH, Lebowitz RL. Lesions of Cowper's ducts and glands in infants and children. Urology. 1978;11:321-325. doi:10.1016/00904295(78)90225-X

3. Bugeja S, Frost A, Ivaz S, Dragova M, Andrich DE, Mundy AR. Syringoceles of Cowper's ducts and glands in adult men. Asian J Androl. 2020;22:129. doi:10.4103/aja.aja_59_19
4. Aguilera Marui C, Brena Zorrilla M. Abscesos para-uretrales gonococicos sin uretritis [Gonococcal para-urethral abscesses without urethritis]. Actas Dermosifiliogr. 1948;39(4):491-493. Spanish.

5. Birnstingl MA, Griffiths JD, Nicol CS, Redmond A. Two cases of perineal fistula following cowperitis. Br J Vener Dis. 1957. doi:10.1136/sti.33.4.246

6. Pepe P, Pepe L, Bonaccorsi A, Panella P, Pennisi M. A complicated case of recurrent Cowper's gland abscess. Arch Ital Urol Androl. 2019;91. doi:10.4081/aiua.2019.3.196.

7. Pietro P, Ludovica P, Astrid B, Paolo P, Michele P. Sepsis secondary to Cowper's gland abscess. Urol Case Rep. 2017;15:48-49. doi:10.1016/j.eucr.2017.09.005

8. NICE guideline [NG51]. Sepsis: recognition, diagnosis and early management. 2016.

9. Dugar S, Choudhary C, Duggal A. Sepsis and septic shock: guideline-based management. Cleve Clin J Med. 2020;87:53-64. doi:10.3949/ccjm.87a.18143

10. Singer M, Deutschman CS, Seymour CW, et al. The third international consensus definitions for sepsis and septic shock (Sepsis-3). JAMA. 2016;315:801. doi:10.1001/jama.2016.0287

11. Rhodes A, Evans LE, Alhazzani W, et al. Surviving sepsis campaign: international guidelines for management of sepsis and septic shock: 2016. Intensive Care Med. 2017. doi:10.1007/s00134-017-4683-6

\section{Publish your work in this journal}

Research and Reports in Urology is an international, peer-reviewed, open access journal publishing original research, reports, editorials, reviews and commentaries on all aspects of adult and pediatric urology in the clinic and laboratory including the following topics: Pathology, pathophysiology of urological disease; Investigation and treatment of urological disease; Pharmacology of drugs used for the treatment of urological disease. The manuscript management system is completely online and includes a very quick and fair peer-review system, which is all easy to use. Visit http://www.dovepress.com/ testimonials.php to read real quotes from published authors. 